\title{
THE ROLE OF BINARITY IN THE EVOLUTION OF PECULIAR RED GIANTS
}

\author{
R.D. McClure \\ Dominion Astrophysical Observatory, National Research Council, Canada
}

\section{INTRODUCTION}

There are several types of Peculiar Red Giants (PRG's) which have enhanced s-process elements and/or carbon, but which cannot be explained by mixing during helium shell-flashing in the late stages of Asymptotic Giant Branch (AGB) evolution. These are the $\mathrm{BaII}, \mathrm{CH}, \mathrm{sgCH}$, and the hotter $\mathrm{R}$-type carbon stars. All these PRG's have absolute magnitudes which range down to zero and fainter. The BaII stars (Bidelman \& Keenan 1951) are G-K giants whose spectra have strong $\mathrm{CN}$ and $\mathrm{CH}$ bands, and in extreme cases bands of $\mathrm{C}_{2}$ become noticeable. In addition they have strong lines of s-process elements such as BaII and SrII. The CH stars (Keenan 1942) are Population II equivalents of the BaII stars, and show similar spectral features, but with weaker metal lines, and usually stronger carbon bands. Bond (1974) introduced a new class of late F and $\mathrm{G}$ stars named the $\mathrm{CH}$ subgiants, which he suggests are fainter than the classical $\mathrm{CH}$ and BaII stars. They appear to be of mixed population (Luck \& Bond 1982), and they are probably subgiants which will eventually evolve up the giant branch to become classical $\mathrm{CH}$ and $\mathrm{BaII}$ stars. The $\mathrm{R}$-type carbon stars, at least the hotter (R0-R4) ones, differ from many of the other peculiar red giants in that they do not show enhanced s-process element abundances. The $\mathrm{R}$ stars have strong carbon bands, but relative to the $\mathrm{N}$-type carbon stars they have less blue and ultraviolet absorption in their spectra. They range in absolute magnitude from near zero, similar to the BaII stars, up to several magnitudes brighter than this.

In 1976, McClure \& Norris (1977) discovered a CH star in the globular cluster M22, and noted that $\omega$ Cen (which also contains CH stars) and M22 possess the common feature that they are very loose clusters for their mass. McClure (1979) speculated that these two clusters along with M55, another low concentration cluster in which a $\mathrm{CH}$ star had been discovered, perhaps contain the $\mathrm{CH}$ stars because these are binary systems. Therefore, an observational program was begun to monitor the radial velocities of faint PRG's to look at the binary frequency among them. The radial-velocity spectrometer on the $1.2 \mathrm{~m}$ telescope coudé at Dominion Astrophysical Observatory (McClure et al. 1985) was used for these observations, giving an accuracy for a velocity of better than $1 \mathrm{~km} \mathrm{~s}^{-1}$. In 1979 , observations were begun of a sample of BaII stars since they tended to be brighter, and a few years later samples of $\mathrm{CH}, \mathrm{R} 0-\mathrm{R} 4$, and $\mathrm{sgCH}$ stars were added to the program. Preliminary results of these programs have been reported by McClure et al. (1980), McClure (1983, 1984a,b), and McClure et al. (1985). The conclusions reached in these papers were that probably all $\mathrm{BaII}$ and $\mathrm{CH}$ stars are binaries, but that the $\mathrm{R}$ stars have a normal binary frequency for giants. The $\mathrm{BaII}, \mathrm{CH}$, and $\mathrm{sgCH}$ stars are too faint to contaminate their own atmospheres by mixing, in the same manner as the stars in the late stages of AGB evolution. It has been suggested widely in the literature, therefore, that they may have been contaminated by mass exchange from a companion which was formerly an AGB star. The latter component of the binary system should now be a white dwarf. However, numerous stars have been observed with IUE (e.g. Böhm-Vitense $e t$ al. 1984; Bond 1984) to look for UV light from white dwarf companions, with only a very few found. 


\section{BINARY FREQUENCY}

Observations have now been obtained over a period of 10 years for the BaII stars, and somewhat less than this for the other types. Relatively good statistics can now be reported on the binary frequency among these stars as follows:

$\begin{array}{cccc}\text { BaII } & \mathrm{CH} & \mathrm{sgCH} & \mathrm{R} \\ 17-18 / 20 & 11 / 12 & 7 / 10 & 4 / 15\end{array}$

where the number of binaries is given relative to the total number in each sample. The conclusions of the preliminary studies mentioned above still stand. The BaII stars and CH stars are almost certainly all binaries. One or two stars in each group not showing velocity variations can be explained by the probability of observing a star pole on. In addition, one of the BaII stars, HD 130255, which has a constant radial velocity, has been shown by Keenan (this conference) not to exhibit the enhanced CN bands characteristic of BaII stars. Keenan has suggested that this may be a dwarf star.

New observations by Jorissen \& Mayor (1988) have now confirmed the binary nature of the BaII stars. In a sample of 27 stars, they have found that 24 show significant velocity variations in their observations which span over three seasons.

Although observations of sgCH stars commenced several years after the other groups, there are now enough observations to say tentatively that they too are all binaries. One or two of the nonvariables may yet turn out to be variable with further observations. Finally, the result still stands that the R0-R4 stars appear to have a binary frequency normal for giant stars. Some different mechanism must be found to explain the high carbon abundances for the $R$ stars. It is important to note that these stars do not show s-process enhancement (Dominy 1984), and therefore it should not be surprising if they are not the result of mass transfer from an AGB star like the other faint PRG's.

\section{THE CASE OF THE S STARS}

Two recent studies have indicated that the $S$ stars may not all be AGB stars which are in the process of helium shell-flashing in the M-MS-S-SC-C sequence as has been suggested widely in the literature. Smith \& Lambert (1988) have found that $38 \%$ (22 out of 58 ) of these s-process enriched stars do not show Tc. Similar results are reported by Little-Marenin (this conference). Since Tc has a half-life of $2 \times 10^{5} \mathrm{yr}$, and this is an s-process element produced in AGB evolution, it would be expected that it should be present in all $\mathrm{S}$ stars. They suggest that those stars not showing Tc in their spectra may be binaries which have undergone mass transfer in the past like the BaII stars, which also lack Tc. Indeed, they find that the space density of these Tc-poor $S$ stars relative to $M$ giants is comparable to the space density, relative to normal $\mathrm{G}$ and $\mathrm{K}$ giants, of the BaII stars.

Secondly, Jorissen \& Mayor (1988) have reported that eight out of a sample of nine S stars have shown velocity variations in observations over three observing seasons. These observations may suggest that a very high percentage of $S$ stars are binaries, contrary to expectations that most are single AGB stars. The evidence for $\mathrm{S}$ stars being in the $\mathrm{M}-\mathrm{S}-\mathrm{C}$ sequence on the AGB is strong, however (see Bessell et al. 1983; Lloyd Evans 1984). The Jorissen \& Mayor result can perhaps be explained by the selection criterion for their stars. Since they picked only photometrically constant $S$ stars for their sample, it is likely that they missed those stars that are in the late stages of AGB evolution. Most nonvariable $S$ stars are hotter, and perhaps these are the ones that are evolved BaII stars with no Tc. Obviously, this problem is ripe for solution within the next year or two. 


\section{ORBITAL ELEMENTS OF BINARY PRG'S}

Webbink (1986) has made a comparison between the orbital eccentricities and mass functions of BaII stars and normal G-K giant stars using the data on seven BaII star orbits available at that time (McClure 1983). He found that the BaII stars have smaller eccentricities on average than a sample of normal $\mathrm{G}-\mathrm{K}$ giant stars. He concluded that there has been some dissipation of the orbit indicating that one of the binary components must, in the past, have filled or nearly filled its Roche Lobe. Because the eccentricities are not zero, however, there must not have been common envelope evolution. Webbink also looked at the distribution of mass functions for the seven BaII stars and concluded that they all have very similar mass-ratios, and for the expected masses for BaII star primaries, the secondary masses should lie between 0.45 AND $0.86 M_{\odot}$, a reasonable range for white dwarfs.

The periods of many of the binary PRG's are very long, but orbits have now been computed for 16 of the BaII stars and $8 \mathrm{CH}$ stars. In order to compare the binary PRG's and normal G-K giant stars, a sample of orbital elements for 43 normal binaries was collected from the work of Griffin (1983, 1984 a,b, 1985 a,b,c, 1986 a,b, 1988 a,b). This sample was limited to those stars which had MK spectral classification indicating giant luminosity and $\mathrm{G}$ or $\mathrm{K}$ spectral type, and orbital periods in the same range (greater than 80 days) as the $\mathrm{BaII}$ and CH stars. Griffin's velocity accuracy is similar to that for the PRG binaries discussed here, and this high accuracy as well as his systematic search and long baseline has minimized the severe biases usually found in binary orbit compilations (Griffin 1985c).

In order to compare the eccentricities of the PRG's and normal giants, their cumulative distributions are plotted in Figure 1. As evident in this figure, the BaII stars have significantly lower eccentricies than the normal giant stars, and the CH stars have even lower eccentricies. As pointed out by Webbink (1986), significantly non-zero eccentricities are very rare among semi-detached binaries. Thus mass transfer or common envelope evolution seems to damp quite strongly any orbital eccentricity.

Fig. 1. The fraction of BaII stars, $\mathrm{CH}$ stars, and normal giant binaries with orbital eccentricities less than a given eccentricity.

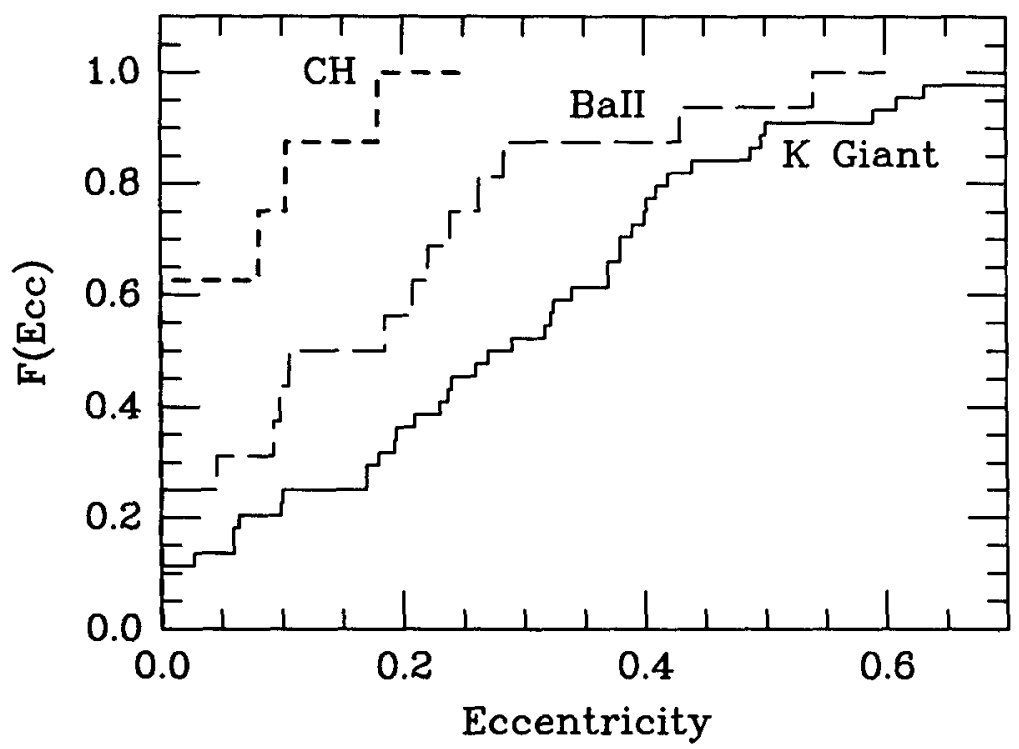


Theoretical studies of tidal dissipation (Zahn 1977), or of the dynamics of mass transfer streams (Piotrowski 1964; Kruszewski 1966) also indicate this. See also the review by Shu $\&$ Lubow (1981). For the case of the $\mathrm{CH}$ stars, one would be hard pressed to say that they are not a sample of binaries with circularized orbits, which suggests common-envelope evolution could have taken place. The BaII stars, however, exhibit decidedly non-zero eccentricities for the most part, which is surprising if they are post-mass-transfer binaries. On the other hand, the fact that they have significantly lower eccentricities than normal stars is clear evidence for dissipation of the original orbital energy in these systems by some mass-transfer mechanism. Two hypotheses have been put forward for this mechanism. The first is tidal dissipation (Webbink 1986), where the companion of the BaII star has, in the past, filled or nearly filled its Roche Lobe. The second mechanism, suggested by Boffin \& Jorissen (1988) to circumvent the problem of rapid circularization of orbits in the case of Roche Lobe overflow, is transfer of mass by a stellar wind and planetary nebula. In this case the system always remains detached, and their model allows eccentric orbits to survive the mass transfer process. On the other hand, the exact effect on orbital eccentricity is uncertain.

Further evidence for mass transfer in BaII and $\mathrm{CH}$ stars comes from an examination of the distribution of mass functions of these systems, as defined by $f(m)=\sin ^{3} i \cdot m_{2}^{3} /\left(m_{1}+m_{2}\right)^{2}$. The cumulative distribution of mass functions for the sample of normal giants, BaII stars and $\mathrm{CH}$ stars are shown in Figure 2. As in the case of the eccentricities, the distributions of mass functions for these three samples is very different. Notice that the BaII stars have considerably lower mass functions than the $\mathrm{CH}$ stars, and that the distribution of mass functions for the normal giants has a very different shape. Whereas the BaII and $\mathrm{CH}$ star distributions rise quickly to a relatively low maximum value, the distribution for the normal giants has an excess of large mass functions.

Fig. 2. The fraction of Ball stars, $\mathrm{CH}$ stars, and normal giant binaries with mass functions less than a given value.

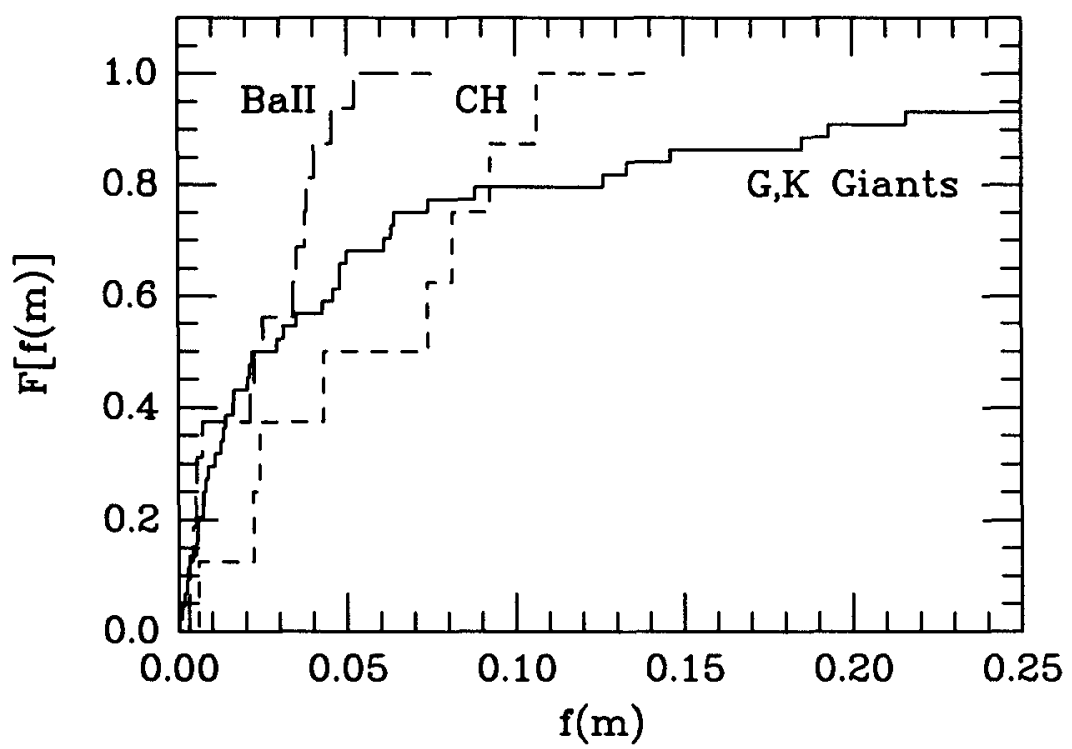


To understand the nature of these differences, let us first examine the cumulative distribution of mass functions expected for a sample of binaries that are homogeneous with respect to mass ratio of the components. In Figure 3, the cumulative distribution is plotted (solid curve) for a sample of binaries which have a constant value of the mass ratio, or true mass function $m_{2}^{3} /\left(m_{1}+m_{2}\right)^{2}=0.04 \mathrm{M}_{\odot}$. This, and all other comparisons described below were calculated by modeling the distribution of the mass function assuming a random distribution of orbital inclinations on the sky. In any real sample of stars, even a homogeneous sample, it would be expected that there would be some dispersion in mass ratio of the components. The long-dashed curve in Figure 3 displays the distribution of mass functions modeled for a sample of stars with true mass-functions near $m_{2}^{3} /\left(m_{1}+m_{2}\right)^{2}=0.04$, but with a small dispersion of 0.01 about this value, as shown by the inset in the bottom right side of the figure. The distribution of mass functions for a similar model with twice the dispersion is shown by the short-dashed curve. Notice that, unlike the distribution of mass functions for normal giants (Figure 2), these models do not exhibit the large values of the mass function. The mass functions for normal giants, as expected, are not distributed like those of a homogeneous sample.

Figure 4 illustrates a model (inset figure) that does fit the cumulative distribution of mass functions for normal giants. The dashed curve in Figure 4 represents the modeled distribution, while the solid lines represent the observed distribution for Griffin's sample. As expected, there is a broad distribution of true mass-functions, representing a sample of binaries with a large spread in masses and mass ratios. In order to reproduce the curved cumulative-distribution for normal giants a majority of the systems have to have small values of the true mass-function (relatively small masses and large mass ratios), but with a tail toward large values of the true mass-function (large and near equal masses). Small variations from this will produce a noticeably poorer fit to the observed distribution.

Fig. 3. The fraction of stars with mass functions less than a given value for models of the true mass-function as shown in the inset figure and for a sample with random distribution of orbital inclinations.

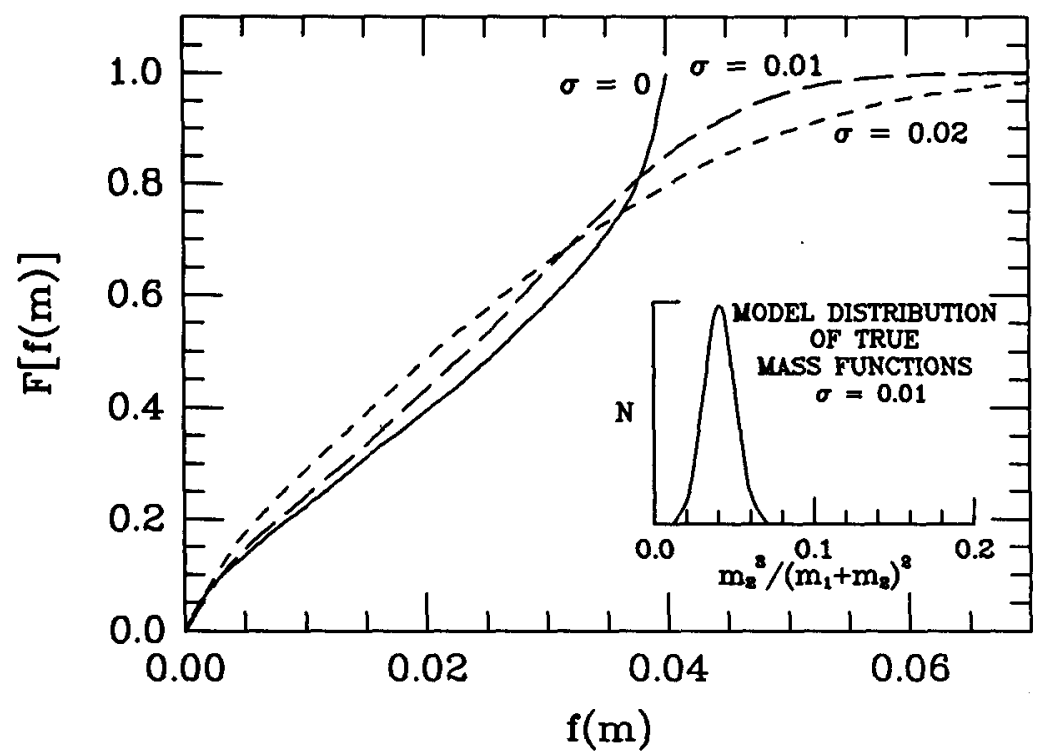


Fig. 4. The fraction of normal giant binaries with mass functions less than a given value (solid lines), and for a model (dashed curve) assuming random orbital inclinations and a distribution of the true mass-function as shown in the inset figure.

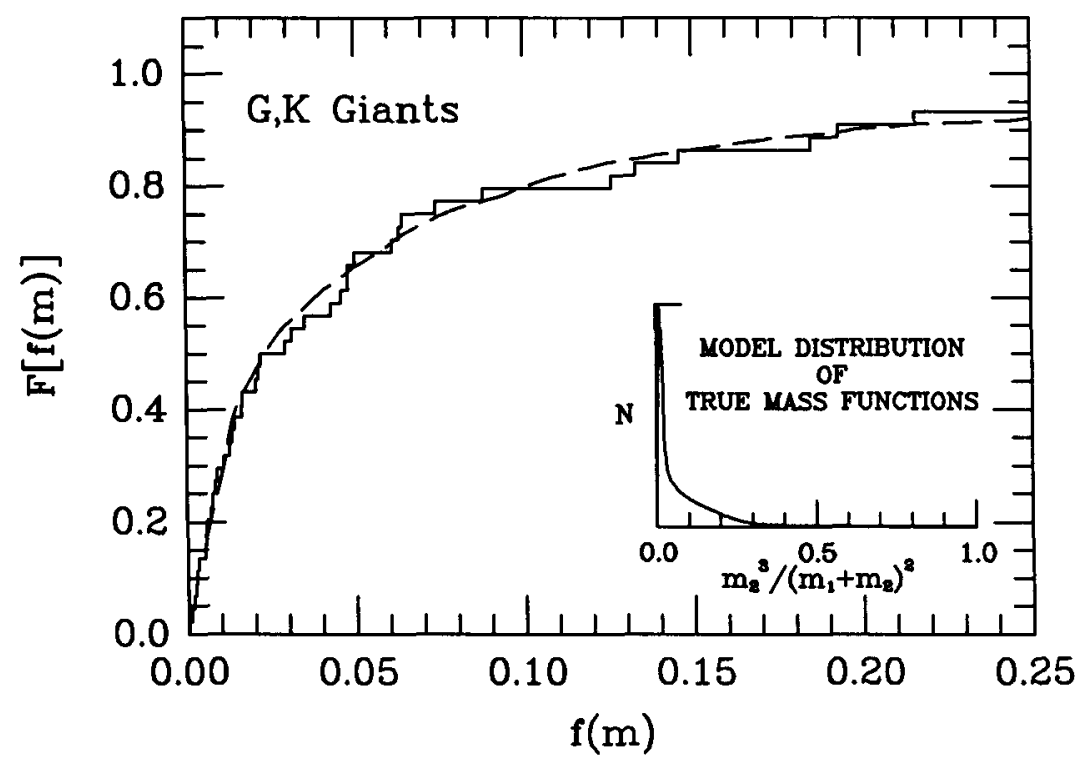

Fig. 5. The fraction of BaII binaries with mass functions less than a given value (solid lines), and for a model (dashed curve) assuming random orbital inclinations and a distribution of the true mass-function as shown in the inset figure.

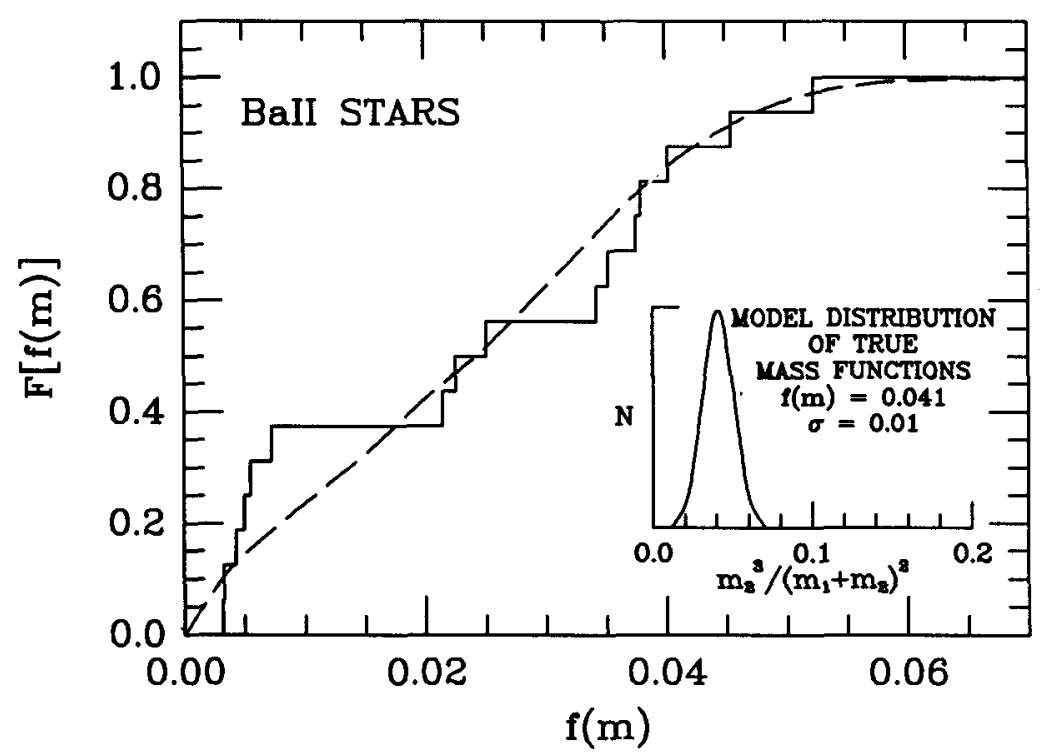


The situation for the $\mathrm{BaII}$ and $\mathrm{CH}$ stars is quite different; the distribution of their mass functions is amazingly like those of homogeneous samples. Figure 5 shows the cumulative distribution of mass functions for the BaII stars, and a model for a sample of stars of true mass-function of $0.041 \mathrm{M}_{\odot}$ and dispersion $\sigma=0.01$ about this value (illustrated in the inset). Within the errors of the rather small sample, the fit of the model to the observed distribution is excellent. The conclusion drawn by Webbink (1986) still stands, therefore, that the masses of the companions to the barium stars must be highly correlated with those of the barium stars themselves, and that it is possible that both are virtually single-valued. A similarily good fit is obtained for the $\mathrm{CH}$ stars (Figure 6) by a model with true massfunction of $0.095 \mathrm{M}_{\odot}$ and dispersion of 0.015 .

BaII stars have been thought to be members of the old disk population based on their kinematics (Eggen 1972), with the confirming evidence of the membership of two BaII stars in an old disk cluster NGC2420 (McClure et al. 1974; Suntzeff \& Smith 1988). Williams (1975) and Hakkila (this conference) suggest, however, that the BaII stars are younger than this, and Catchpole et al. (1977) suggest that there may be an age spread in the sample. The masses of the BaII stars probably lie in the range of about $1.0 \mathrm{M}_{\odot}$ (from the discussion of kinematics by Eggen 1972) to $<3 \mathrm{M}_{\odot}$ (as suggested from the highest masses found from visual binary membership by Culver \& Ianna $(1976,1980)$ and Culver et al. (1977), and by the discussion of kinematics by Hakkila). An estimate for the average mass of a BaII star of about $1.5 \mathrm{M}_{\odot}$, therefore, cannot be far wrong, this relatively low value being suggested because of the secure evidence of membership in the old disk cluster. Given this mass for the primaries of the BaII stars and the value found from orbits for the true mass function of $0.041 \mathrm{M}_{\odot}$, the mass calculated for the companions is $m_{2}=0.56 \mathrm{M}_{\odot}$, a quite reasonable value for the mass of a white dwarf.

Fig. 6. The fraction of $\mathrm{CH}$ star binaries with mass functions less than a given value (solid lines), and for a model (dashed curve) assuming random orbital inclinations and a distribution of the true mass-function as shown in the inset figure.

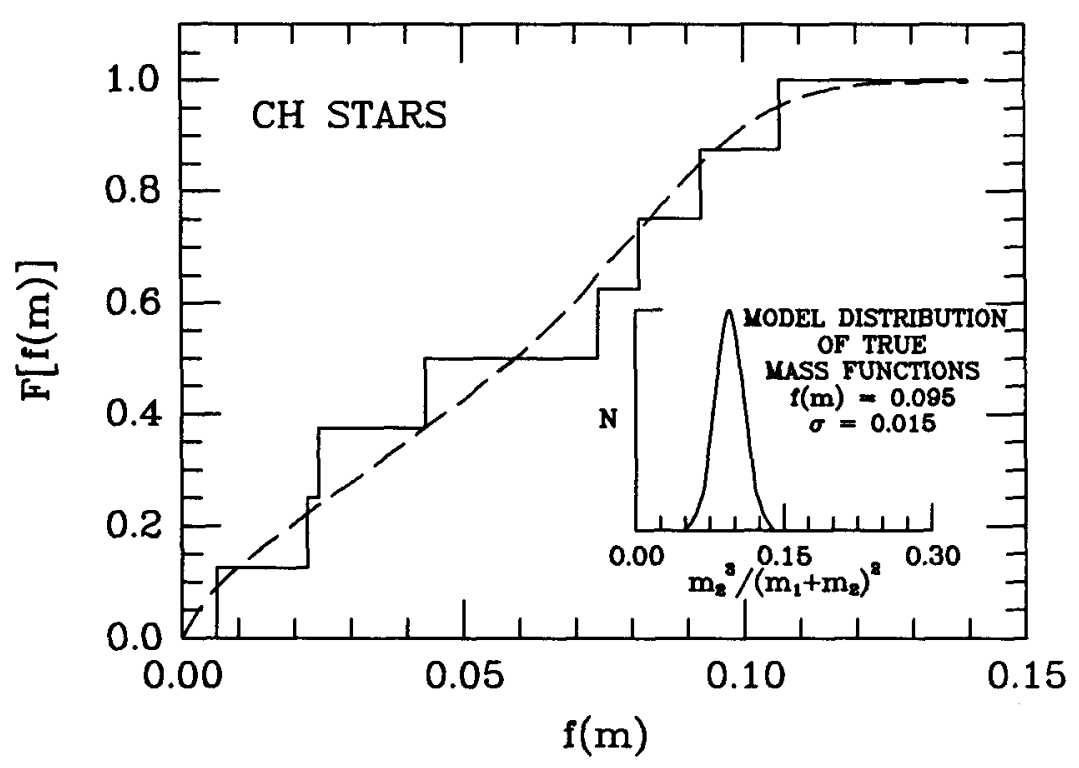


$\mathrm{CH}$ stars, being members of Population II, likely have masses of $0.8 \pm 0.1 \mathrm{M}_{\odot}$. The corresponding masses of the companions, given the value of the true mass-function of 0.095 $\mathrm{M}_{\odot}$ found above, is again $0.56 \mathrm{M}_{\odot}$. The fact that the secondary masses for both BaII stars and $\mathrm{CH}$ stars comes out the same is a coincidence, given the uncertainty in the masses of the primaries. The similarity of the resultant masses for the secondaries to the value we expect for white dwarfs, however, is quite surprising, especially considering the different stellar populations, and different mass ratios of the binaries in these two samples. This gives us confidence that the mass-transfer hypothesis for contamination of the atmospheres of the low-luminosity PRG's is very plausible.

\section{QUESTIONS FOR DISCUSSION}

Although binarity among BaII and $\mathrm{CH}$ stars helps to explain how the atmospheres of some of the PRG's may have been contaminated, there are numerous questions raised by this discovery. Some of these that come to mind are the following.

1) Where do the $\mathbf{R}$-type carbon stars fit into the picture of the evolution of PRG's? These stars differ from other PRG's in that they do not appear to have enhanced s-process elements. The hotter members of this class are not bright enough to be on the asymptotic giant branch where helium shell-flashing is occurring, and the contamination of the stellar atmosphere with carbon cannot be blamed on mass transfer in a binary system because they appear to have a normal binary frequency. The peculiar abundances in the $\mathrm{R}$ stars must be explained by a different evolutionary process than those used to explain any other stars discussed at this conference. It is likely that the helium core flash is responsible (see discussion by Dominy 1984), but more theoretical work must be done to show how this works.

2) What is the status of the S stars? Smith and Lambert (1988) and Little-Marenin (this conference) suggest that $\mathrm{S}$ stars which do not exhibit Tc lines (about $38 \%$ of the sample) may be mass-transfer binaries, with the BaII stars being the precursors. Jorissen and Mayor (1988), however, have found that almost all of the S stars they have observed have variable radial velocities. Are there two sequences of $S$ stars, and why do Jorissen and Mayor seem to find almost all S stars are binaries? They picked photometrically nonvariable stars for their sample, and as they mention, this may bias the sample towards evolved $\mathrm{BaII}$ stars as opposed to stars in the $\mathrm{M}-\mathrm{S}-\mathrm{C}$ sequence.

3) If all $\mathrm{BaII}$ and $\mathrm{CH}$ stars are binaries with white dwarf companions, as the orbital parameters discussed here strongly suggest, why is there so little evidence for white dwarfs found in the IUE observations of their ultraviolet spectra? Can the lack of white dwarfs be explained by their having cooled to luminosities below the detection limit, and is this telling us something about time scales for the evolution and binary mass-transfer process?

4) If binarity is necessary for the BaII star phenomenon, why are the only two cluster BaII stars known found in the same cluster (NGC2420)? Also, why is one of these two in such a peculiar position in the color-magnitude diagram, off the giant branch towards the blue (see McClure 1984a)?

5) Why are the CH stars that have been found in globular clusters located mainly in those clusters with low central concentration as well as in the very loose dwarf spheroidal galaxies? If this correlation is not just a coincidence, are more binaries formed to begin with in the low concentration clusters, or is it a result of disruption of binaries in concentrated clusters? 
I wish to thank Dr. Andy Woodsworth, who has contributed significantly to the program of measuring velocities of PRG's during the last year.

\section{REFERENCES}

Bessell, M.S., Wood, P.R., \& Lloyd Evans, T. (1983). Mon. Not. Roy. Astron. Soc., $202,59$. Bidelman, W.P., \& Keenan, P.C. (1951). Astrophys. J., 114, 473.

Boffin, H.M.J., \& Jorissen, A. (1988). preprint.

Böhm-Vitense, E., Nemec, J.M., \& Proffitt, C. (1984). Astrophys. J., 278, 726.

Bond, H.E. (1974). Astrophys. J., 194, 95.

Bond, H.E. (1984). Future of Ultraviolet Astronomy based on Six Years of I.U.E. Research,

N.A.S.A. Conf. Publ. No. 2349, p. 289.

Catchple, R.M., Robertson, B.S.C., \& Warren, P.R. (1977). Mon. Not. Roy. Astron. Soc., $181,391$.

Culver, R.B., \& Ianna, P.A. (1976). Publ. Astron. Soc. Pacific, $88,41$.

Culver, R.B., \& Ianna, P.A. (1980). Publ. Astron. Soc. Pacific, 92, 829.

Culver, R.B., Ianna, P.A., \& Franz, O.G. (1977). Publ. Astron. Soc. Pacific, 89, 397.

Dominy, J.F. (1984). Astrophys. J. Suppl., 55, 27.

Eggen, O.J. (1972). Mon. Not. Roy. Aston. Soc., 159, 403.

Griffin, R.F. (1983). Observatory, 103, 273.

Griffin, R.F. (1984a). Observatory, 104, 6.

Griffin, R.F. (1984b). Observatory, 104, 268.

Griffin, R.F. (1985a). Observatory, 105, 9.

Griffin, R.F. (1985b). Observatory, 105, 128.

Griffin, R.F. (1985c). In Interacting Binaries, ed. P.P. Eggleton \& J.E. Pringle, p. 1.

Dordrecht: Reidel.

Griffin, R.F. (1986a). Observatory, 106, 35.

Griffin, R.F. (1986b). Observatory, 106, 108.

Griffin, R.F. (1988a). Observatory, 108, 17.

Griffin, R.F. (1988b). Observatory, 108, 90.

Jorissen, A., \& Mayor, M. (1988). Astron. Astrophys., 198, 187.

Keenan, P.C. (1942). Astrophys. J., 96, 101.

Kruszewski, A. (1966). Adv. Astron. Astrophys., 4, 233.

Lloyd Evans, T. (1984), Mon. Not. Roy. Astron. Soc., 208, 447.

Luck, R.E., \& Bond, H.E. (1982). Astrophys. J., 259, 792.

McClure, R.D. (1979). Mem. Soc. Astron. Italiano, 50, 15.

McClure, R.D. (1983). Astrophys. J., 268, 264.

McClure, R.D. (1984a). Publ. Astron. Soc. Pacific, $\underline{96}, 117$.

McClure, R.D. (1984a). Astrophys. J. (Letters), 280, L31.

McClure, R.D., Fletcher, J.M., Grundmann, W.A., \& Richardson, E.H. (1985). In I.A.U.

Colloq. No. 88, Stellar Radial Velocities, ed. A.G.D. Philip, \& D.W.

Latham, p. 49, Schenectady: L. Davis Press.

McClure, R.D., Fletcher, J.M., \& Nemec, J.M. (1980). Astrophys. J. (Letters)., 238, L35.

McClure, R.D., Forrester, W.T., \& Gibson, J. (1974). Astrophys. J., 189, 409.

McClure, R.D., \& Norris, J. (1977). Astrophys. J. (Letters), 217, L101.

Piotrowski, S.L. (1964). Acta Astron., 14, 251.

Shu, F.H., \& Lubow, S.H. (1981). Ann. Rev. Astron. Astrophys., 19, 277.

Smith, V.V., \& Lambert, D.L. (1988). Astrophys. J. in press.

Suntzeff, N.B., \& Smith, V.V. (1988). Astron. J., 93, 359.

Webbink, R.F. (1986). In Critical Observations versus Physical Models for Close Binary

Systems (Proc. Beijing Colloq. on Close Binary Systems), ed. K.-C. Leung,

\& D.S. Zhai, in press, New York: Gordon \& Breach.

Williams, P.M. (1975). Mon. Not. Roy. Astron. Soc., 170, 343.

Zahn, J.P. (1977). Astron. Astrophys., 57, 383. 